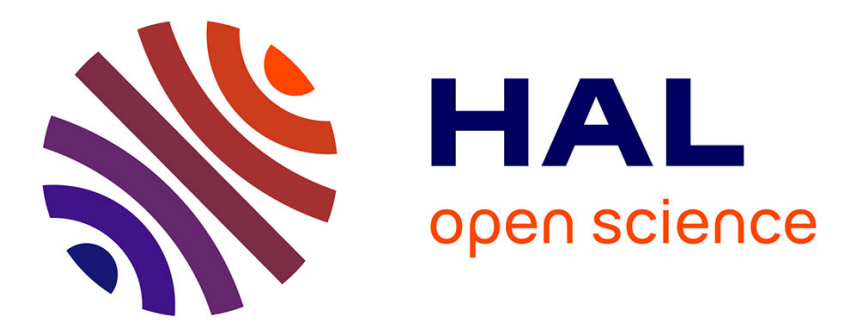

\title{
Probability box as a tool to model and control the effect of epistemic uncertainty in multiple dependent competing failure processes
}

Qingyan Zhang, Zhiguo Zeng, Enrico Zio, Rui Kang

\section{- To cite this version:}

Qingyan Zhang, Zhiguo Zeng, Enrico Zio, Rui Kang. Probability box as a tool to model and control the effect of epistemic uncertainty in multiple dependent competing failure processes. Applied Soft Computing, 2017, 56, pp.570-579. 10.1016/j.asoc.2016.06.016 . hal-02447683

\section{HAL Id: hal-02447683 \\ https://hal.science/hal-02447683}

Submitted on 24 Feb 2020

HAL is a multi-disciplinary open access archive for the deposit and dissemination of scientific research documents, whether they are published or not. The documents may come from teaching and research institutions in France or abroad, or from public or private research centers.
L'archive ouverte pluridisciplinaire HAL, est destinée au dépôt et à la diffusion de documents scientifiques de niveau recherche, publiés ou non, émanant des établissements d'enseignement et de recherche français ou étrangers, des laboratoires publics ou privés. 


\title{
Probability box as a tool to model and control the effect of epistemic uncertainty in multiple dependent
} competing failure processes

\author{
Qingyuan Zhang ${ }^{1}$, Zhiguo Zeng ${ }^{2 *}$, Enrico Zio ${ }^{2,3}$ and Rui Kang ${ }^{1}$ \\ ${ }^{1}$ Center for Resilience and Safety of Critical Infrastructures, Beihang University, Beijing, China \\ ${ }^{2}$ Chair on Systems Science and Energetic Challenge, Fondation Electricité de France (EDF), CentraleSupelec, \\ Universite' Paris-Saclay, Chatenay-Malabry, France \\ ${ }^{3}$ Energy Department, Politecnico di Milano, Italy \\ zhangqingyuan@buaa.edu.cn, zhiguo.zeng@centralesupelec.fr (*), enrico.zio@ecp.fr, kangrui@buaa.edu.cn
}

\section{Abstract}

Engineering components and systems are often subject to multiple dependent competing failure processes (MDCFPs). MDCFPs have been well studied in literature and various models have been developed to predict the reliability of MDCFPs. In practice, however, due to the limited resource, it is often hard to estimate the precise values of the parameters in the MDCFP model. Hence, the predicted reliability is affected by epistemic uncertainty. Probability box (P-box) is applied in this paper to describe the effect of epistemic uncertainty on MDCFP models. A dimension-reduced sequential quadratic programming (DRSQP) method is developed for the construction of P-box. A comparison to the conventional construction method shows that DRSQP method reduces the computational costs required for P-box constructions. Since epistemic uncertainty reflects the unsureness in the predicted reliability, a decision maker might want to reduce it by investing resource to more accurately estimate the value of each model parameter. A two-stage optimization framework is developed to allocate the resource among the parameters and ensure that epistemic uncertainty is reduced in a most efficient way. Finally, the developed methods are applied on a real case study, a spool valve, to demonstrate their validity. 


\section{Introduction}

Engineering components and systems are often subject to multiple processes that lead to their failures. Usually, these processes compete to cause failures and are dependent in various ways. Hence, they are referred to as multiple dependent failure processes (MDCFPs) [1]. In practice, most MDCFPs involve both degradation processes and random shocks [2]. MDCFPs have been well studied in literature and various models have been developed to predict the reliability of MDCFPs. A typical example is [3], in which Peng et al. considered a MDCFP where failure can be caused by either a degradation process or a random shock process. The two failure processes were dependent since when a random shock arrives, an abrupt increase to the degradation process is caused. A similar model can be found in [4], where degradation is modeled by a diffusion process and the random shocks are assumed to follow a Poisson process. Wang and Pham [5] modeled MDCFP in a similar way, within a framework for defining the optimal imperfect preventive maintenance policy. Li and Pham [6, 7] developed a reliability model and an inspection-maintenance model for multistate degrading systems, considering two degradation processes and a shock process. Keedy and Feng [8] applied Peng's approach ([3]) to model a stent, where the degradation process is modeled by a Physics of Failure (PoF) model. In a recent paper by Lin et al. [9], the degradation process is modeled by a continuous-time, semi Markov process and the shock process is modeled using a homogeneous Poisson process.

Most existing MDCFP models assume that the precise values of the model parameters are known to the modeler. In practice, however, due to the limited resource, it is often difficult to precisely estimate the model parameters. Hence, the reliability predicted by the MDCFP models are affected by epistemic uncertainty [10]. The effect of epistemic uncertainty should be accounted for in MDCFP models.

In literature, there are various approaches to describe epistemic uncertainty, e.g. Bayesian theory [11], evidence theory $[12,13]$, possibility theory [14], fuzzy theory [15], probability box (P-box) [16], etc. The major differences among these theories are the way that the incomplete knowledge is interpreted and mathematically described. For example, applying Bayesian theory implies that one can represent our incomplete knowledge as prior probability distributions $[17,18]$, evidence theory expresses incomplete knowledge by identifying basic probability assignments (BPA) $[12,13]$, possibility theory relies on possibility distributions (or membership functions) to describe the state of knowledge [19, 20], probability box (P-box) expresses the incomplete knowledge based on intervals or bounds of probability distributions [21, 22]. Among them, P-box is natural to engineers and easier to implement in practice. Therefore, in this paper, we use P-box to describe epistemic uncertainty in MDCFP models. 
A P-box comprises a pair of upper and lower cumulative density functions (CDFs), in which the real CDF is bounded. P-boxes have been widely applied in many fields to solve the problems associated with epistemic uncertainty, such as engineering [23], biology [24], environmental science [25], etc. An early implementation of P-box can date back to the work of Walley and Fine [26], where they constructed a probability box to model the imprecision in probability estimations. Wolfenson and Fine [27] used upper and lower probabilities to support Bayesian-like decision making. Ferson et al. [16] used P-boxes to handle both variability (aleatory uncertainty) and ignorance (epistemic uncertainty) in safety assessments. In the field of reliability, Karanki et al. [21] applied P-box to evaluate the probability of system failure under the influence of epistemic uncertainty. Xiao et al. [28] proposed a unified method to perform sensitivity analysis reliability for structural systems by combining the P-box, first-order reliability method (FORM) and Monte Carlo simulation (MCS). Luis et al. [29] applied P-box to the analysis of polynomial systems subject to parameter uncertainties. Zhang developed interval Monte Carlo Simulation (IMCS) method [22], interval importance sampling method [30] and quasi-Monte Carlo method [31] for finite element-based structural reliability assessment based on P-boxes. In order to reduce the calculation cost of IMCS method, Yang et al. [32] introduced a hybrid method based on P-box, where the true limit state equation is approximated by a surrogate model, to evaluate the reliability of structures.

In existing P-box method, the epistemic uncertainty is propagating by calculating the Cartesian products of the input parameters and their P-boxes (see [21] for example). More specifically, to calculate the reliability of a time-varying system which is described by P-boxes, the time interval under investigation is discretized into several subintervals and the maximum and minimum reliability is searched using numerical optimization methods in each subinterval [33]. A major drawback of these uncertainty propagation methods is that their computational costs grow as the number of model parameters increases. In this paper, we develop a dimension-reduced SQP method, which uses gradient information to reduce the required computational costs. reduce it by investing resource to more accurately estimate the value of each model parameter. A two-stage optimization framework is developed to allocate the resource among the parameters and ensure that epistemic uncertainty is reduced in a most efficient way. The contribution of this paper is summarized as follows: processes (MDCFPs);

Since epistemic uncertainty reflects the unsureness in the predicted reliability, a decision maker might want to 
- an optimization model is developed to control the effect of epistemic uncertainty in MDCFP.

The remainder of this paper is organized as follows. Section 2 presents how to quantify the epistemic uncertainty on a MDCFP model using P-box. The DRSQP method is developed in Section 3 for the construction of the P-box. In Section 4, a two-stage optimization framework is developed for the optimal reduction of epistemic uncertainty. A real case study on a sliding spool is conducted in Section 5 to demonstrate the developed methods.

6 Finally, the paper is concluded in Section 6 with a discussion on possible future research directions.

\section{Representing epistemic uncertainty using P-boxes}

In this section, we briefly review the concept of P-box in subsection 2.1, and then, apply it in subsection 2.2 to describe epistemic uncertainty on MDCFP models.

\subsection{Preliminaries on P-boxes}

A P-box comprises of an upper and a lower cumulative density function (CDF), denoted by $\left[\underline{F}_{x}, \bar{F}_{x}\right]$. The actual CDF of a random variable $x$, denoted by $F_{x}(x)$, is bounded in the area:

$$
\underline{F}_{x} \leq F_{x}(x) \leq \bar{F}_{x}
$$

14 Hence, the distance between the upper and lower CDFs represents the amount of epistemic uncertainty.

A simple illustration of a P-box is given in Figure 1. Suppose $x$ is a random variable following a normal distribution with the variance $\sigma^{2}=1$. However, due to the limited time and resource, the precise value of the mean value cannot be estimated accurately. The only information we have is that, the mean value lies in the interval of $[0,3]$. The epistemic uncertainty in $x$ can, then, be described by a P-box in Figure 1. It is easy to show that the actual CDF lies in the bounded area constructed by $\underline{F}_{x}$ and $\bar{F}_{x}$.

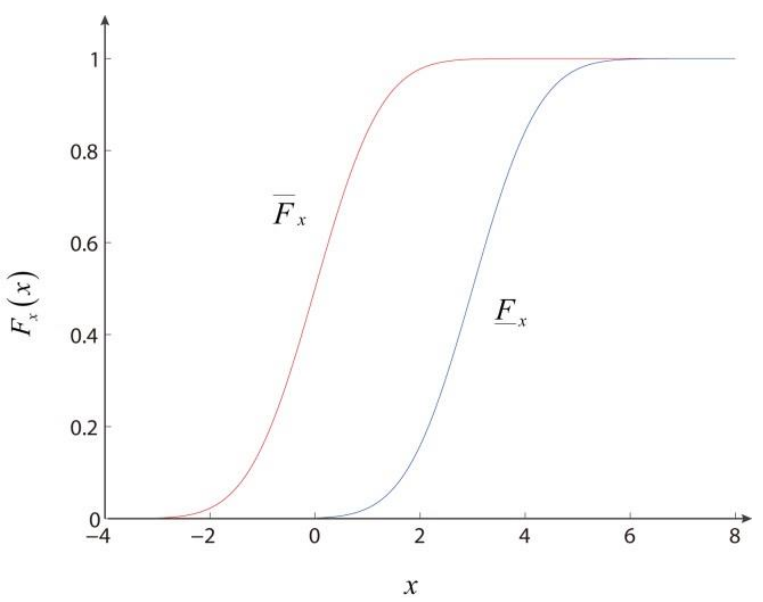




\subsection{Describing epistemic uncertainties in MDCFP by P-boxes}

For most MCDFP, reliability is modeled as an explicit function of $t$ [3]:

$$
R(t)=f_{\text {MDCFP }}(t ; \mathbf{x})
$$

4 where $\mathbf{x}=\left[x_{1}, x_{2}, \cdots, x_{n}\right]^{T}$ is a vector of parameters in the reliability model. Due to epistemic uncertainty, the precise values of $\mathbf{x}$ is not known to us. Often, the parameters are estimated by experts in the form of intervals:

$$
\mathbf{x} \in I_{\mathbf{x}}=\left[x_{1, L}, x_{1, U}\right]^{T} \times\left[x_{2, L}, x_{2, U}\right]^{T} \times \cdots \times\left[x_{n, L}, x_{n, U}\right]^{T} .
$$

Such effect of epistemic uncertainty, is, then, described by constructing a P-box for the estimated reliability:

$$
R_{L}(t) \leq R(t) \leq R_{U}(t)
$$

9 where $R_{U}(t)$ and $R_{L}(t)$ are the upper and lower bounds of the reliability function, respectively. In this paper, the P-box in (4) is called a reliability box. Given available information, all the possible reliability estimates are 11 bounded in the reliability box.

The area covered by the reliability box, denoted by $A_{R B}$, reflects the amount of epistemic uncertainty. When

13 knowledge on the failure processes accumulates, more information is possessed so that more precise estimates on the values of $\mathbf{x}$ could be made. As a result, the intervals for $\mathbf{x}$ shrink and the reliability box shrinks accordingly.

15 In an ideal situation that there is no epistemic uncertainty, the precise values of $\mathbf{x}$ is known to us. Therefore, the reliability box reduces to a single curve.

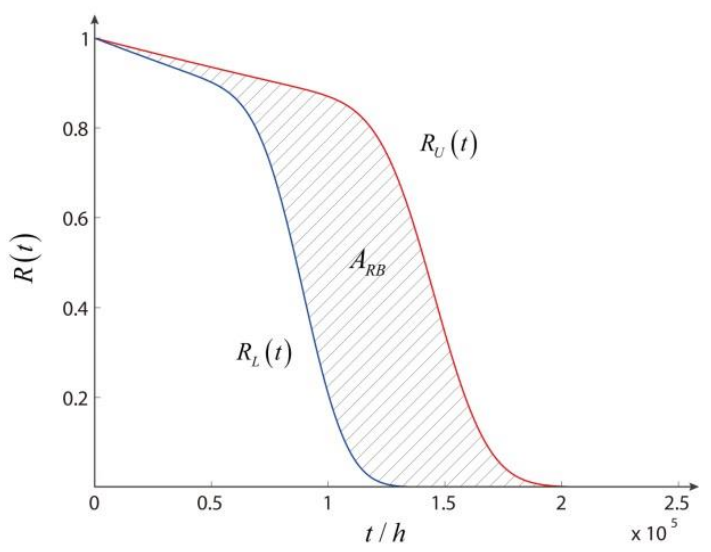

Figure 2 An illustration of the reliability box

Suppose $R_{L}(t)$ and $R_{U}(t)$ denote the upper and lower bounds of reliability at time $t$, respectively. Then, 


$$
A_{R B}=\int_{0}^{\infty}\left[R_{U}(\xi)-R_{L}(\xi)\right] \mathrm{d} \xi
$$

For a given MDCFP model, the form of the reliability function is fixed (as shown in (2)). Therefore, the $R_{U}(t)$ and $R_{L}(t)$ in (5) are determined by the $I_{\mathrm{x}}$ in (3). Hence, (5) can be viewed as a function of $I_{\mathrm{x}}$ :

$$
A_{R B}=h\left(I_{\mathbf{x}}\right)
$$

Note that the mean time to failure (MTTF) can be determined by:

$$
\mathrm{MTTF}=\int_{0}^{\infty} R(\xi) \mathrm{d} \xi
$$

Given a reliability box, we have $R_{L}(\xi) \leq R(\xi) \leq R_{U}(\xi)$. Therefore, the upper and lower limits of MTTF are:

$$
\begin{aligned}
& \operatorname{MTTF}_{U}=\int_{0}^{\infty} R_{U}(\xi) \mathrm{d} \xi, \\
& \operatorname{MTTF}_{L}=\int_{0}^{\infty} R_{L}(\xi) \mathrm{d} \xi .
\end{aligned}
$$

From (5) and (8), we have,

(1)

$$
A_{R B}=\mathrm{MTTF}_{U}-\mathrm{MTTF}_{L} .
$$

Equation (9) explains the physical meaning of $A_{R B}$ : it can be regarded as the difference between the estimated upper and lower MTTF values in presence of epistemic uncertainty. We present a numerical algorithm (Algorithm 1) to calculate $A_{R B}$ when a reliability box $\left[R_{L}, R_{U}\right]$ is given.

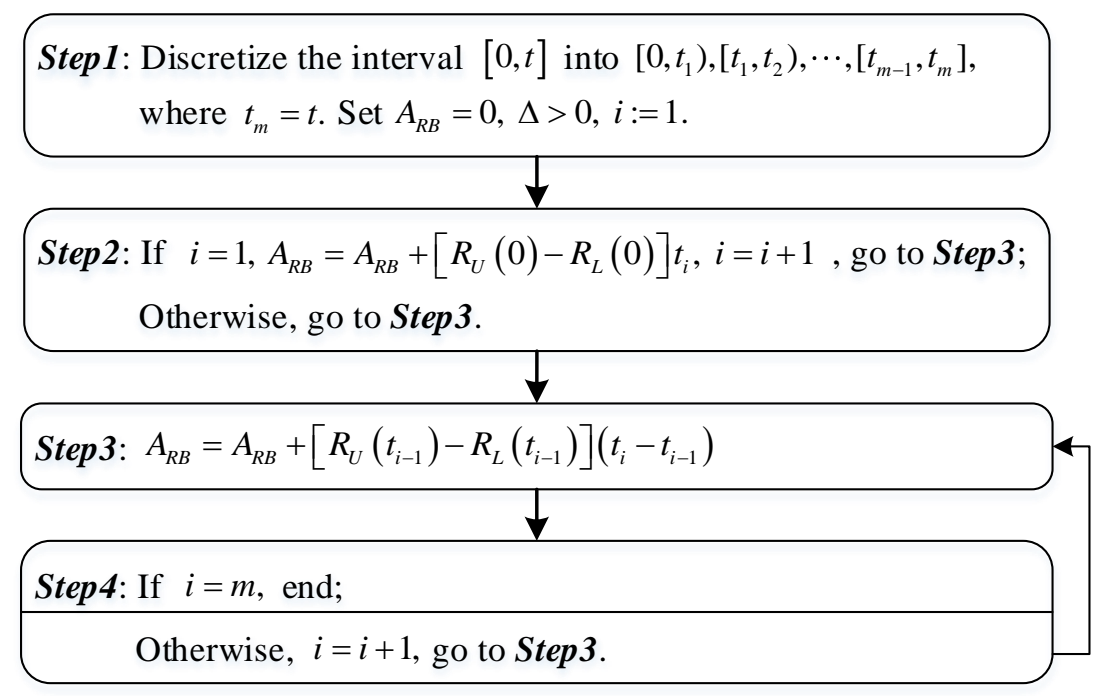

Figure 3 Main steps to calculate $A_{\mathrm{RB}}($ Algorithm 1)

\subsection{A numerical example}

In this subsection, we demonstrate how to use the reliability box to describe epistemic uncertainty using a 
numerical example. Consider a ceramic capacitor whose reliability can be predicted by

$$
R(t)=e^{-\lambda(\mathbf{x}) t}=e^{-\frac{t}{1000} e^{-9.48+0.01759 x_{1}+7.017 x_{2}}}
$$

3 where $\lambda(\mathbf{x})$ denotes the failure rate and is given by

$$
\lambda(\mathbf{x})=\frac{1}{1000} e^{-9.48+0.01759 x_{1}+7.017 x_{2}}
$$

where $x_{1}$ denotes the operating temperature and $x_{2}$ denotes the ratio of operating voltage and nominal voltage [34].

In practice, it is often difficult to obtain the precise values of the voltage and temperature. Normally, they are estimated by experts and given in the form of intervals. Suppose the estimated intervals for $x_{1}$ and $x_{2}$ are $x_{1} \in[35,45]$ and $x_{2} \in[0.719,0.781]$, respectively. Since the reliability function in (10) is monotonic with respect to both $x_{1}$ and $x_{2}$, a reliability box can be easily constructed to describe the epistemic uncertainty, as shown in uncertainty.

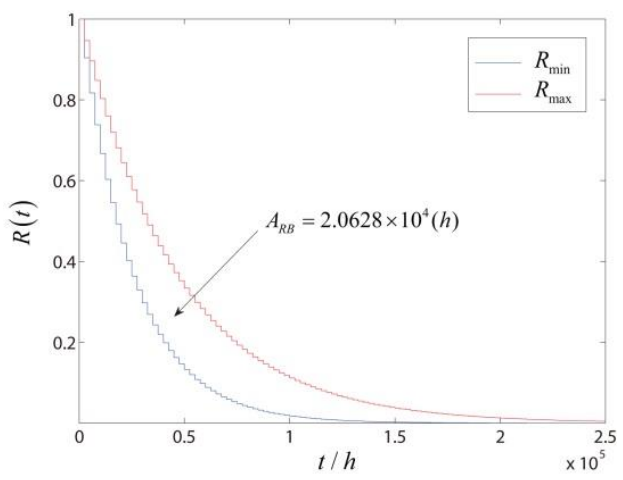

Figure 4 The reliability box of the ceramic capacitor

\section{Dimension-reduced SQP methods}

In this section, we develop a dimension-reduced sequential quadratic programming (DRSQP) method to reduce the computational costs required in the construction of P-boxes. In subsection 3.1, we introduce the conventional outer-discretization method as a benchmark method for P-box constructions. Then, the DRSQP method is developed in subsection 3.2. The computational efficiencies of the DRSQP and benchmark methods are compared in subsection 3.3 via a numerical case study.

\subsection{SQP-based uncertainty propagation using P-boxes}

For time-varying systems, the outer-discretization method is often used to propagate uncertainties described 
by P-boxes [33]. In the outer discretization method, the time interval is discretized into several subintervals and the maximum and minimum reliability values are searched using numerical optimization methods in each subinterval. Based on different numerical optimization methods, various outer discretization methods can be developed. In this subsection, we introduce an outer discretization method which is based on Sequential Quadratic Programming $5 \quad(\mathrm{SQP})$.

SQP is one of the best known methods to solve constrained optimization problems [35]:

$$
\begin{aligned}
& \min f(\mathbf{x}), \\
& \text { s.t. } c_{i}(x)=0, i \in \mathrm{E} \\
& c_{i}(x) \geq 0, i \in \mathrm{I}
\end{aligned}
$$

where $\mathbf{x} \in \mathfrak{R}^{n}, c_{i}(x)$ denotes the $i$ th constraint of the problem, and $\mathrm{E}$ and I denote the equality constraint set and inequality constraint set, respectively.

At each iteration of the SQP method, a new design point $\left(\mathbf{x}_{k+1}, \boldsymbol{\lambda}_{k+1}\right)$ is determined so that the KKT conditions (Karush-Kuhn-Tucker conditions) are satisfied [35]:

$$
\begin{array}{ll}
\nabla_{x} L\left(\mathbf{x}_{k+1}, \lambda_{k+1}\right)=0 & \\
c_{i}\left(\mathbf{x}_{k+1}\right)=0, & i \in \mathrm{E}, \\
c_{i}\left(\mathbf{x}_{k+1}\right) \geq 0, & i \in \mathrm{I}, \\
\lambda_{k+1}^{(i)} \geq 0, & i \in \mathrm{I}, \\
\lambda_{k+1}^{(i)} c_{i}\left(\mathbf{x}_{k+1}\right)=0, & i \in \mathrm{E} \bigcup \mathrm{I} .
\end{array}
$$

13 where $\lambda$ is the Lagrangian multiplier of the Lagrange function $L(\mathbf{x}, \lambda)=f(\mathbf{x})-\sum_{i} \lambda_{i} c_{i}(\mathbf{x}), \lambda_{k}^{(i)}$ denotes the $i$ th 14 element in $\lambda_{k}$ and $\mathbf{x}_{k+1}=\mathbf{x}_{k}+\mathbf{d}_{k}$. In order to determine $\mathbf{d}_{k}$, a quadratic programming (QP) is conducted, which according to [35], is equivalent to the KKT conditions in (13):

$$
\begin{array}{ll}
\min _{\mathbf{d}} & q(\mathbf{d})=\frac{1}{2} \mathbf{d}^{T} W\left(\mathbf{x}_{k}\right) \mathbf{d}+g^{T}\left(\mathbf{x}_{k}\right) \mathbf{d}+f\left(\mathbf{x}_{k}\right) \\
\text { s.t. } & c_{i}\left(\mathbf{x}_{k}\right)+a_{i}\left(\mathbf{x}_{k}\right)^{T} d=0, i \in \mathrm{E} \\
& c_{i}\left(\mathbf{x}_{k}\right)+a_{i}\left(\mathbf{x}_{k}\right)^{T} d \geq 0, i \in \mathrm{I}
\end{array}
$$

In (14), $a_{i}\left(\mathbf{x}_{k}\right)=\nabla c_{i}\left(\mathbf{x}_{k}\right), f\left(\mathbf{x}_{k}\right)$ is second derivable with respect to $\mathbf{x}$ and 


$$
\begin{aligned}
& g\left(\mathbf{x}_{k}\right)=\nabla_{\mathbf{x}} L\left(\mathbf{x}_{k}, \lambda_{k}\right)= {\left[\frac{\partial L}{\partial x_{k, 1}^{(1)}}, \frac{\partial L}{\partial x_{k}^{(2)}}, \cdots, \frac{\partial L}{\partial x_{k}^{(n)}}\right]^{T}, } \\
& W\left(\mathbf{x}_{k}\right)=\nabla_{\mathbf{x}}^{2} L\left(\mathbf{x}_{k}, \lambda_{k}\right)=\left[\begin{array}{cccc}
\frac{\partial^{2} L}{\partial\left(x_{k}^{(1)}\right)^{2}} & \frac{\partial^{2} L}{\partial x_{k}^{(1)} \partial x_{k}^{(2)}} & \cdots & \frac{\partial^{2} L}{\partial x_{k}^{(1)} \partial x_{k}^{(n)}} \\
\frac{\partial^{2} L}{\partial x_{k}^{(2)} \partial x_{k}^{(1)}} & \frac{\partial^{2} L}{\partial\left(x_{k}^{(2)}\right)^{2}} & \cdots & \frac{\partial^{2} L}{\partial x_{k}^{(2)} \partial x_{k}^{(n)}} \\
\vdots & \vdots & & \vdots \\
\frac{\partial^{2} L}{\partial x_{k}^{(n)} \partial x_{k}^{(1)}} & \frac{\partial^{2} L}{\partial x_{k}^{(n)} \partial x_{k}^{(2)}} & \cdots & \frac{\partial^{2} L}{\partial\left(x_{k}^{(n)}\right)^{2}}
\end{array}\right]
\end{aligned}
$$

2 denote the gradient and Hessian matrix of $L\left(\mathbf{x}_{k}, \boldsymbol{\lambda}_{k}\right)$, respectively. In each iteration, $W\left(\mathbf{x}_{k}\right)$ is corrected

3 according to DFP (Davidon-Fletcher- Powell) or BFGS (Broyden-Fletcher- Goldfarb-Shanno) methods [35].

To guarantee the global convergence of the method, a line search is required to determine the step size $\alpha_{k}$.

5 Let $\mathbf{x}_{k+1}=\mathbf{x}_{k}+\alpha_{k} \mathbf{d}_{k}$ and let the penalty function $P_{L_{1}}(\mathbf{x}, \boldsymbol{\sigma})$ be [36]:

6

$$
P_{L_{1}}(\mathbf{x}, \boldsymbol{\sigma})=f(\mathbf{x})+\sum_{i} \sigma_{i}\left|\bar{c}_{i}(\mathbf{x})\right|
$$

where

8

$$
\begin{aligned}
& \bar{c}_{i}(\mathbf{x})= \begin{cases}c_{i}(\mathbf{x}), & i \in \mathrm{E} \\
\min \left\{0, c_{i}(\mathbf{x})\right\}, & i \in \mathrm{I}\end{cases} \\
& \sigma_{k}^{(i)}= \begin{cases}\lambda_{k}^{(i)}, & k=0 \\
\max \left\{\left|\lambda_{k+1}^{(i)}\right|, \frac{1}{2}\left(\sigma_{k-1}^{(i)}+\left|\lambda_{k+1}^{(i)}\right|\right)\right\}, & k \geq 1\end{cases}
\end{aligned}
$$

9 Line searches should be conducted until an $\alpha_{k}$ which satisfies Armijo law is found [35].

The implementation procedures of the SQP method are summarized in Algorithm 2 below. 


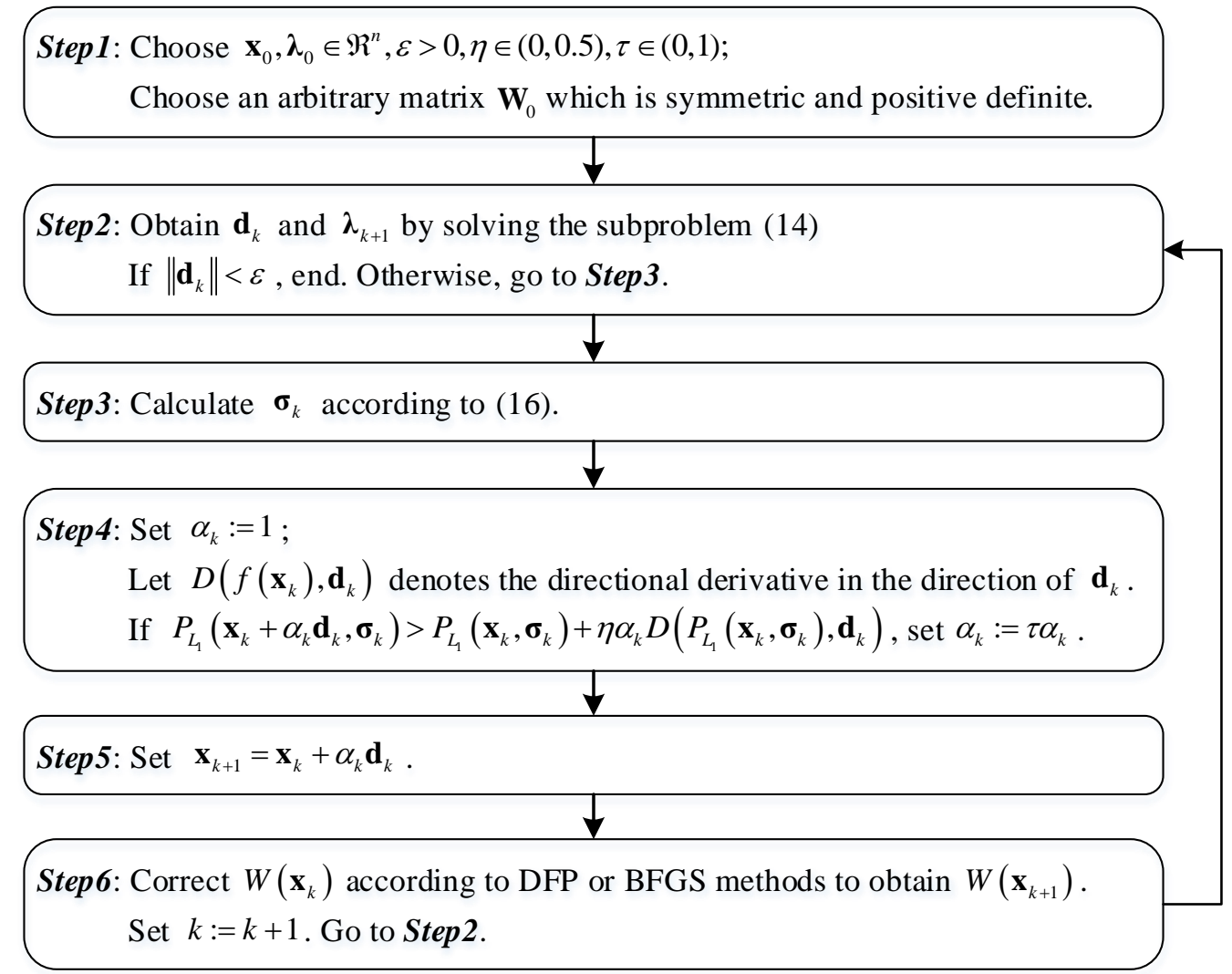

Figure 5 Main steps for the SQP method (Algorithm 2) [35]

Based on the SQP method, the outer-discretization method can be implemented in a double-loop procedure. In 4 the outer loop, the interval $[0, t]$ is discretized into $m$ subintervals, $\left[0, t_{1}\right),\left[t_{1}, t_{2}\right), \cdots,\left[t_{m-1}, t_{m}\right]$, where $t_{m}=t$. In 5 the inner loop, SQP methods are used to find the maximum and minimum values for $R\left(t_{i}\right)$ when $\mathbf{x}$ varies in the 6 interval of $I_{\mathrm{x}}$ :

$$
\begin{aligned}
& \min R\left(t_{i}\right)=f_{M C D F P}\left(t_{i} ; \mathbf{x}\right) \\
& \text { s.t. } \mathbf{x} \in I_{\mathbf{x}} . \\
& \max R\left(t_{i}\right)=f_{M C D F P}\left(t_{i} ; \mathbf{x}\right) \\
& \text { s.t. } \mathbf{x} \in I_{\mathbf{x}} .
\end{aligned}
$$

9 In (17) and (18), the constraints $I_{\mathrm{x}}$ take the form expressed in (3). The $R_{L}\left(t_{i}\right)$ and $R_{U}\left(t_{i}\right), i=1,2, \cdots, m$

10 provide a pointwise approximation of the reliability box.

\section{$11 \quad 3.2 . \quad$ The DRSQP method}

The computation costs of applying the double-loop procedure increase as the dimension of $\mathbf{x}$ increases.

13 Based on the available information on the gradients, the dimension of the optimization problem in (17) and (18) 14 could be reduced before the SQP methods are applied. To do this, we first prove Theorem 1. 


\section{Theorem 1:}

Suppose $y=f(\mathbf{x})$ is a multivariate function defined in $I_{\mathbf{x}}$, where $I_{\mathbf{x}}$ is defined by (3). Further, $f(\mathbf{x})$ is continuous in $I_{\mathbf{x}}$ and $g(\mathbf{x})$ are continuous in $I_{\mathbf{x}}-\delta I_{\mathbf{x}}$. If $\forall \mathbf{x} \in I_{\mathbf{x}}$,

$$
\frac{\partial f(\mathbf{x})}{\partial x_{i}}>0, \frac{\partial f(\mathbf{x})}{\partial x_{j}}<0
$$

Then, we have

$$
\begin{aligned}
& f\left(\mathbf{x}_{i, L}^{*}\right) \leq f\left(\mathbf{x}_{i}\right), \\
& f\left(\mathbf{x}_{i, U}^{*}\right) \geq f\left(\mathbf{x}_{i}\right) .
\end{aligned}
$$

where $\mathbf{x}_{i, L}^{*}=\left[x_{1}, x_{2}, \cdots, x_{i, L}, x_{i+1}, \cdots, x_{n}\right]^{T}, \mathbf{x}_{i, U}^{*}=\left[x_{1}, x_{2}, \cdots, x_{i, U}, x_{i+1}, \cdots, x_{n}\right]^{T}, \mathbf{x}_{i}=\left[x_{1}, x_{2}, \cdots, x_{i}, \cdots, x_{n}\right]^{T}$, and

$$
\begin{aligned}
& f\left(\mathbf{x}_{j, L}^{*}\right) \leq f\left(\mathbf{x}_{i}\right), \\
& f\left(\mathbf{x}_{j, U}^{*}\right) \geq f\left(\mathbf{x}_{i}\right) .
\end{aligned}
$$

where $\mathbf{x}_{j, L}^{*}=\left[x_{1}, x_{2}, \cdots, x_{j, U}, x_{j+1}, \cdots, x_{n}\right]^{T}, \mathbf{x}_{j, U}^{*}=\left[x_{1}, x_{2}, \cdots, x_{j, L}, x_{j+1}, \cdots, x_{n}\right]^{T}, \mathbf{x}_{j}=\left[x_{1}, x_{2}, \cdots, x_{j}, \cdots, x_{n}\right]^{T}$.

\section{Proof:}

Here we only prove the first inequality in (19) and the others can be proved in a similar way. Let $\mathbf{x}^{*}=\left[x_{1}, x_{2}, \cdots, x_{i, L}, x_{i+1}, \cdots, x_{n}\right]^{T}$, and we assume $\exists \mathbf{x}_{0} \in I_{\mathbf{x}}$, s.t. $f\left(\mathbf{x}^{*}\right)>f\left(\mathbf{x}_{0}\right)$.

From the Mid-value theorem, there is at least one $\mathbf{x}_{1} \in I_{\mathbf{x}}$ so that

$$
f\left(\mathbf{x}^{*}\right)-f\left(\mathbf{x}_{0}\right)=g^{T}\left(\mathbf{x}_{1}\right) \cdot\left(\mathbf{x}^{*}-\mathbf{x}_{0}\right)
$$

So we have $g^{T}\left(\mathbf{x}_{1}\right) \cdot\left(\mathbf{x}^{*}-\mathbf{x}_{0}\right) \leq 0$, i.e. $f\left(\mathbf{x}^{*}\right)-f\left(\mathbf{x}_{0}\right) \leq 0$, which contradicts the assumption. Thus, the theorem is proved.

Let $\mathbf{x}_{\min }^{*}, \mathbf{x}_{\max }^{*}$ denote the value of $\mathbf{x}$ when $f(\mathbf{x})$ takes its minimum and maximum value, respectively, so that $f\left(\mathbf{x}_{\text {min }}^{*}\right) \leq f(\mathbf{x}), f\left(\mathbf{x}_{\text {max }}^{*}\right) \geq f(\mathbf{x}), \forall \mathbf{x} \in I_{\mathbf{x}}$. According to Theorem 1, if $\forall \mathbf{x} \in I_{\mathbf{x}}, \partial f(\mathbf{x}) / \partial x_{i}>0$, then we have $\mathbf{x}_{\min }^{*,(i)}=x_{i, L}, \mathbf{x}_{\max }^{*,(i)}=x_{i, U}$; if $\partial f(\mathbf{x}) / \partial x_{j}<0$, then we have $\mathbf{x}_{\min }^{*,(j)}=x_{i, U}, \mathbf{x}_{\max }^{*,(j)}=x_{i, L}$. These elements can be removed from the optimization problems (17) and (18), in order to reduce the computational costs. This fact motivates the dimension-reduced SQP method (DRSQP), whose main steps are summarized in Figure 6. 


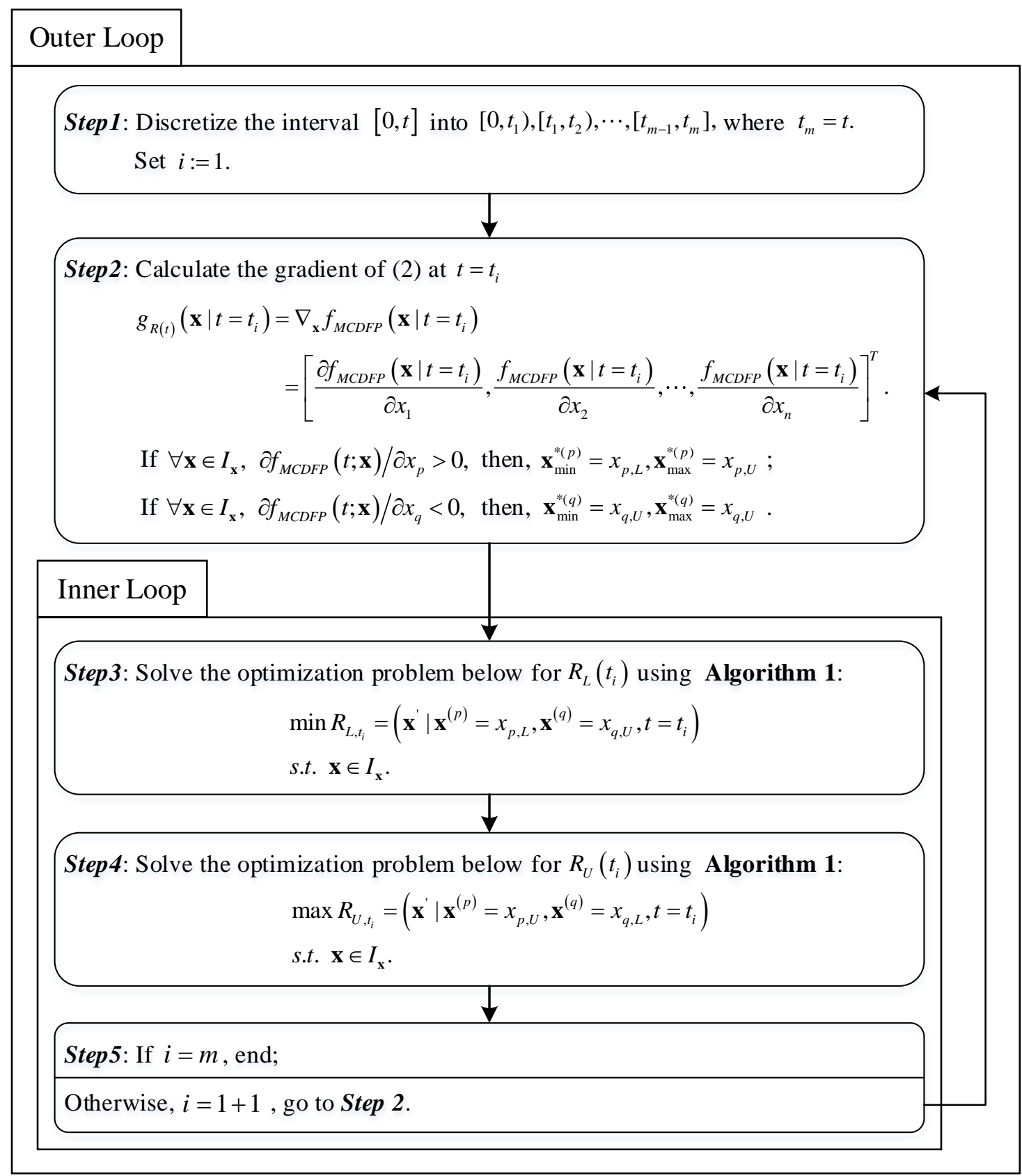

Figure 6 Main steps for the DRSQP method (Algorithm 3)

\subsection{A case study}

In this subsection, we apply the developed DRSQP method to a Micro-Electro-Mechanical System (MEMS) (adapted from [3]) to analyze the effect of epistemic uncertainty on the MDCFP model while, at the same time, we demonstrate the computational efficiency of the DRSQP method. The MEMS system experiences two dependent competing failure processes: (i) soft failure caused by continuous degradation, and (ii) hard failure due to random shocks. These two failure processes are competing, since either of them can lead to the failure of the device, and dependent, since the arrival of random shocks brings an abrupt change to the normal degradation process [3]. A 
reliability model is developed in [3] to describe the MDCFP:

$$
\begin{aligned}
& P_{L}=P\left(W_{i}<D\right)=\Phi\left(\frac{D-\mu_{W}}{\sigma_{W}}\right), i=1,2, \ldots, \infty \\
& P\left(X_{S}(t)<H\right)=P(X(t)+S(t)<H)=\sum_{i=0}^{\infty} \Phi\left(\frac{H-\left(\mu_{\beta} t+\varphi+i \mu_{Y}\right)}{\sqrt{\sigma_{\beta}^{2} t^{2}+i \sigma_{Y}^{2}}}\right) \frac{e^{-\lambda t}(\lambda t)^{i}}{i !}
\end{aligned}
$$

$$
\begin{aligned}
R(t) & =\sum_{i=0}^{\infty}\left(\prod_{j=0}^{i} P\left(W_{j}<D\right)\right) P\left(X_{s}(t)<H\right) \\
& =\sum_{i=0}^{\infty} \Phi^{i}\left(\frac{D-\mu_{W}}{\sigma_{W}}\right) \cdot \Phi\left(\frac{H-\left(\mu_{\beta} t+\varphi+i \mu_{Y}\right)}{\sqrt{\sigma_{\beta}^{2} t^{2}+i \sigma_{Y}^{2}}}\right) \frac{e^{-\lambda t}(\lambda t)^{i}}{i !}
\end{aligned}
$$

3 where $P_{L}$ denotes the probability of system surviving the shock load, $W_{i}$ denotes the size of the $i$ th shock load, and $W_{i} \sim N\left(\mu_{W}, \sigma_{W}^{2}\right), D$ denotes the threshold for hard failures, $X_{S}(t)$ denotes the total volume at $\mathrm{t}$ due to both degradation and shock, $H$ denotes the threshold for soft failures, $X(t)$ denotes the degradation volume at $\mathrm{t}$ and $X(t)=\varphi+\beta t, S(t)$ denotes the cumulative shock damage size at $\mathrm{t}$ and $S(t)=\sum_{i} Y_{i}, \lambda$ denotes the

\begin{tabular}{|c|c|c|c|c|}
\hline \multicolumn{2}{|c|}{ Parameters } & \multirow{2}{*}{$\begin{array}{c}\text { Lower Limit } \\
0.001\end{array}$} & \multirow{2}{*}{$\begin{array}{c}\text { Upper Limit } \\
0.0015\end{array}$} & \multirow{2}{*}{$\begin{array}{l}\text { Midpoint } \\
0.00125\end{array}$} \\
\hline & $H\left(\mu m^{3}\right)$ & & & \\
\hline & $D(G p a)$ & 1.4 & 1.6 & 1.5 \\
\hline & $\lambda$ & $2 \times 10^{-5}$ & $3 \times 10^{-5}$ & $2.5 \times 10^{-5}$ \\
\hline & $\varphi$ & $-2.5 \times 10^{-5}$ & $2.5 \times 10^{-5}$ & 0 \\
\hline \multirow{2}{*}{$\beta$} & $\mu_{\beta}\left(\mu m^{3}\right)$ & $8 \times 10^{-9}$ & $9 \times 10^{-9}$ & $8.5 \times 10^{-9}$ \\
\hline & $\sigma_{\beta}\left(\mu m^{3}\right)$ & $5.5 \times 10^{-10}$ & $6.5 \times 10^{-10}$ & $6.0 \times 10^{-10}$ \\
\hline \multirow{2}{*}{ Y } & $\mu_{r}\left(\mu m^{3}\right)$ & $0.5 \times 10^{-4}$ & $1.5 \times 10^{-4}$ & $1.0 \times 10^{-4}$ \\
\hline & $\sigma_{Y}\left(\mu m^{3}\right)$ & $1.5 \times 10^{-5}$ & $2.5 \times 10^{-5}$ & $2.0 \times 10^{-5}$ \\
\hline \multirow{2}{*}{$W$} & $\mu_{w}(G p a)$ & 1.1 & 1.3 & 1.2 \\
\hline & $\sigma_{W}(G p a)$ & 0.15 & 0.25 & 0.2 \\
\hline
\end{tabular}
arrival rate of random shocks, and $\varphi, \beta, Y$ are normally distributed variables.

Table 1 Interval of parameters given by experts

9

There are ultimately 10 parameters relating to the reliability function. We ask experts to estimate their values, shown as the interval $I_{\mathrm{x}}$ in Table 1. To implement the DRSQP method, the gradients of (21) are calculated first, as given in the Appendix. Then, the reliability box can be constructed according to Algorithm 3. As a comparison, we also construct the reliability box using the SQP method without dimension reduction. The computational costs of the two methods are compared by evaluations to the reliability model, which are tabulated in Table 3 . It can be seen 
that the DRSQP method requires less function evaluations. This is because, according to the Appendix, the first seven parameters can be removed from the optimization model based on Theorem 1. Hence, the computational costs are greatly reduced.

Table 2 Comparison of SQP method and DRSQP method

\begin{tabular}{cc}
\hline Method & Function Count \\
\hline SQP & 7642 \\
DRSQP & 2131
\end{tabular}

5 Furthermore, from Figure 7 it can be seen that the result obtained from the DRSQP method is more accurate than 6 that from the SQP method, since the SQP method sometimes fails to identify the global maxima or minima.

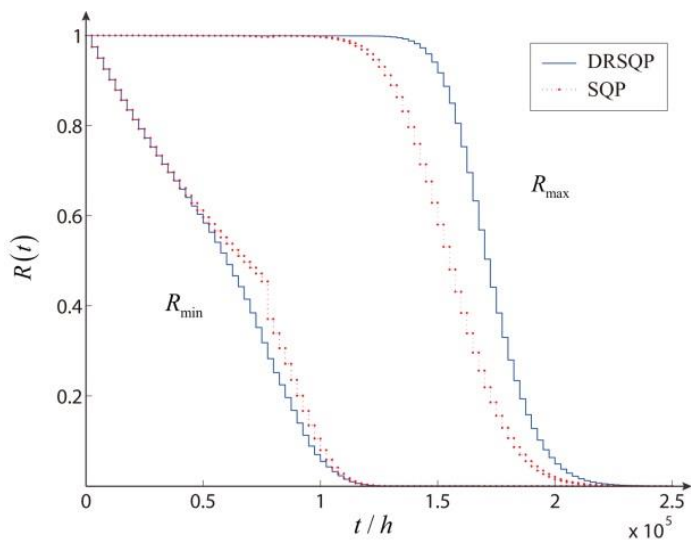

Figure 7 Reliability boxes comparison between DRSQP and SQP methods

Based on the reliability box constructed from the DRSQP method, the $A_{R B}$ can be determined by Algorithm 1 to quantify epistemic uncertainty. In this case study, Algorithm 1 yields $A_{R B}=1.1601 \times 10^{5}$ (h). As shown in (9), the physical meaning of $A_{R B}$ is the difference between the estimated upper and lower MTTF, which reflects the influence of epistemic uncertainty on reliability estimations.

\section{Optimal reduction of epistemic uncertainty}

In this section, we develop an optimization model to reduce the effect of epistemic uncertainty on the MDCFP model. The method is presented in subsection 4.1, followed by a case study in subsection 4.2 to illustrate its application.

\subsection{The model}

According to (9), $A_{R B}$ measures the difference between the upper and lower estimated MTTF. As MTTF is widely used to measure reliability, $A_{R B}$ can be used to quantify the imprecision in reliability evaluations caused by epistemic uncertainty. In practice, it is often required to limit the imprecision to an acceptable level, i.e., 
where

where $A_{R B, r e q}$ is the requirement on the precision of reliability estimations. From (2) and (3), we can see that in order to control $A_{R B}$, we have to reduce the width of the $I_{\mathbf{x}}$ in (3). However, reducing the width of $I_{\mathbf{x}}$ requires the investment of time and resource for acquiring additional knowledge. Hence, the problem of controlling epistemic uncertainty can be formulated as follows.

$$
\begin{aligned}
& \min _{\mathbf{w}} c=C(\mathbf{w}) \\
& \text { s.t. } A_{R B} \leq A_{R B, r e q} .
\end{aligned}
$$

$$
\begin{aligned}
& A_{R B}=h\left(I_{\mathbf{x}}\right), \\
& I_{\mathbf{x}}=\left[x_{1, L}, x_{1, U}\right]^{T} \times\left[x_{2, L}, x_{2, U}\right]^{T} \times \cdots \times\left[x_{n, L}, x_{n, U}\right]^{T}, \\
& x_{i, L}=-\frac{w_{i}}{2}+\frac{x_{0, L}^{(i)}+x_{0, U}^{(i)}}{2}, 1 \leq i \leq n, \\
& x_{i, U}=\frac{w_{i}}{2}+\frac{x_{0, L}^{(i)}+x_{0, U}^{(i)}}{2}, 1 \leq i \leq n .
\end{aligned}
$$

9 In (23), $\mathbf{w}=\left[w_{1}, w_{2}, \cdots, w_{n}\right]^{T}$ is the decision variable, representing the upper and lower bounds for each element in

$10 I_{\mathbf{x}}$, and $w_{i}=x_{i, U}-x_{i, L}, i=1,2, \cdots, n$, where $x_{i, U}$ and $x_{i, L}$ are the upper and lower bounds for the $i$ th element in

$11 \mathbf{x}$. In (24), $x_{0, L}^{(i)}$ and $x_{0, U}^{(i)}$ are the upper and lower limits of the $i$ th element of the initial estimate on $\mathbf{x}$,

12 respectively. The objective function $C(\mathbf{w})$ represents the cost incurred by reducing the $\mathbf{w}$ and should be determined based on engineering experiences. Due to the complexity in the form of $C(\mathbf{w})$ and $h(\cdot)$, the

14 optimization model might be solved by numerical methods, e.g., the genetic algorithm (GA) [37].

\subsection{A case study}

In this subsection, we combine the GA and SQP method to control the effect epistemic uncertainty in the MEMS device discussed in subsection 3.3. As discussed in subsection 3.3, given the available information, the constructed reliability box leads to $A_{R B}=1.1601 \times 10^{5}$ (h), which represents the imprecision in the initial reliability estimates. Suppose that the requirement on epistemic uncertainty is $A_{R B, r e q}=2 \times 10^{4}(\mathrm{~h})$, and the cost incurred by

20 reducing the width of the $I_{\mathbf{x}}$ is:

$$
c=C(\mathbf{w})=\sum_{i=1}^{n} \frac{1}{c_{i} \cdot w_{i}+a_{i}}
$$


where, $c_{i}>0, i=1,2, \cdots, n$ are cost coefficients associated with each $w_{i}, a_{i}$ is a coefficient measuring the

2 difficulty in reducing epistemic uncertainty in $x_{i}$ : when all the $w_{i} \mathrm{~s}$ are reduced to zero, the required cost is $1 / a_{i}$.

3 The two coefficients can be estimated by analyst based on the difficulty in determining the value of the

4 corresponding parameter. In this case study, we assume $c_{i}=1 / 10 w_{0}^{(i)}$ and $a_{i}=0.01$ respectively, where

$5 \quad w_{0}^{(i)}=x_{0, L}^{(i)}-x_{0, U}^{(i)}$.

6 The solution of the model comprises of two stages. In the first stage, an initial attempt is conducted using GA,

7 whose result is used in the second stage as the initial values of the SQP. In the second stage, SQP method is used to 8 improve the search for optima. The optimal solutions from the two stages are tabulated in Table 3.

Table 3 The optimal solutions from the two stages

\begin{tabular}{c}
\multirow{2}{*}{ Parameters } \\
\cline { 2 - 7 }
\end{tabular}

10

Figure 8 shows the reliability box after the application of the model. Table 4 summarizes the relative reduction

12 of the $i$ th element in $\mathbf{x}$, denoted by $I_{i}=w_{0}^{(i)} / w_{i}^{*}$. It is worth noting that the relative reductions are not equally

13 distributed in the elements, indicating an uneven allocation of the resource. The reason for this is that the sensitivity

14 of the elements on the predicted reliability is different. Take the variables $H$ and $\lambda$ as an example. Figure 9

15 illustrates the difference in the sensitivities of the two variables. It is clear that $H$ is more sensitive than $\lambda$.

16 Hence, to more efficiently reduce the influence of epistemic uncertainty, rather than $\lambda$, more resource should be

17 invested to reduce the width of the P-box in $H$, which is in accordance with the results in Table 4 . The

18 optimization scheme developed in (23) can automatically allocate resource among the model parameters, based on 
their sensitivities.

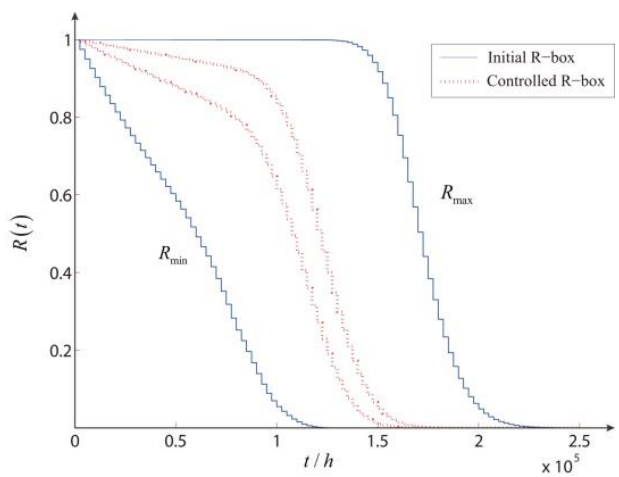

Figure 8 Reliability boxes before and after controlling

Table 4 Resources allocation among the model parameters

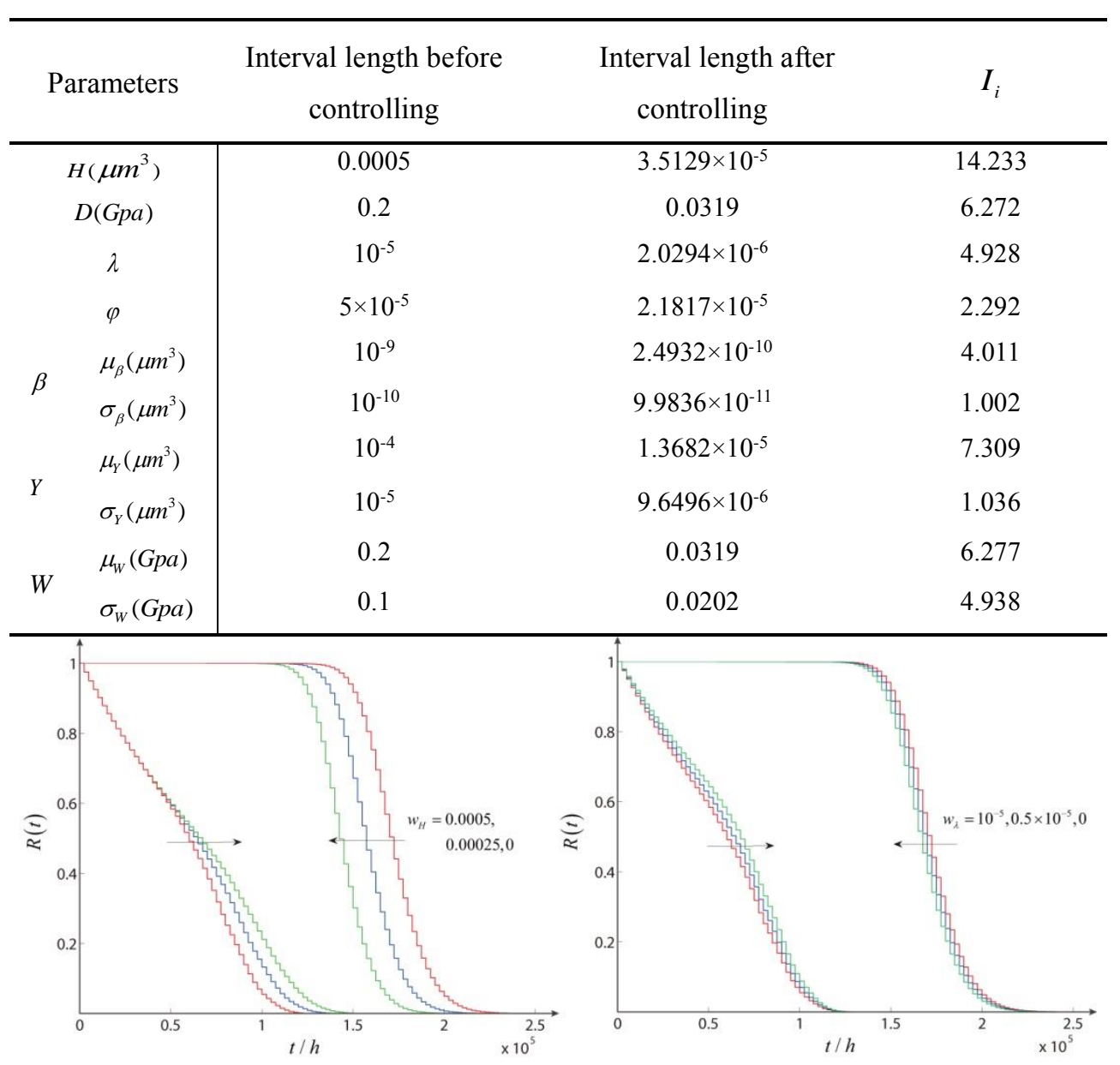

Figure 9 Sensitivity analysis of reliability box on $H$ and $\lambda$

\section{5. Application}

9 In this section, we apply the developed methods to model and control epistemic uncertainty on a real case study,

10 a sliding spool, to further demonstrate the applicability of the developed methods. Failure of a sliding spool is 
primarily caused by two failure mechanisms: (1) wear due to the friction between the spool and the sleeve, and (2) clamping stagnation caused by the pollutant in the hydraulic oil and wear debris [38]. Clamping stagnation can be caused by two different processes, i.e., immediate stagnation and cumulative stagnation. Immediate stagnation happens when a particle whose diameter is larger than the gap between the sleeve and the spool appears. Cumulative stagnation is caused by filer cakes formulated by small particles, as shown in Figure 10.

According to [39], the wear process can be described by a degradation process model and the clamping stagnation can be represented using a random shock model. Therefore, failure occurs whenever one of the three following events happens: (1) excessive wear, where the degradation volume due to continuous wear exceeds degradation threshold; (2) immediate stagnation, where a shock whose magnitude exceeds fatal shock threshold occurs; (3) cumulative stagnation, where the cumulative damage given rise to shocks exceeds damage shock threshold [40]. In this case, we assume the three events are independent, as shown in Figure 11.

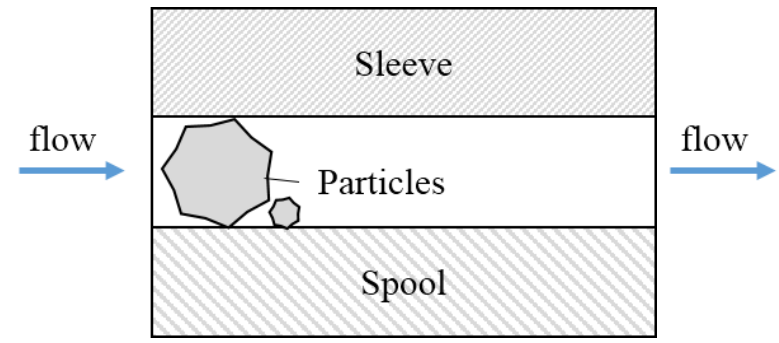

(a) Immediate stagnation

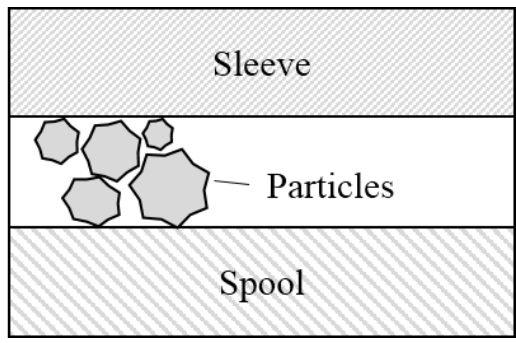

(b) Cumulative stagnation

Figure 10 Two failure mechanisms leading to clamping stagnation [39]

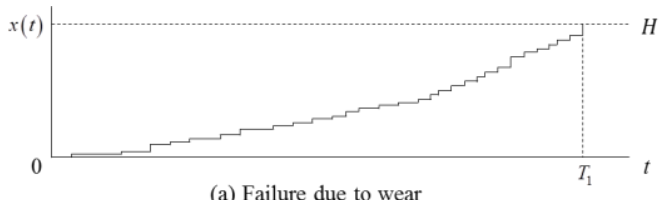

(a) Failure due to wear

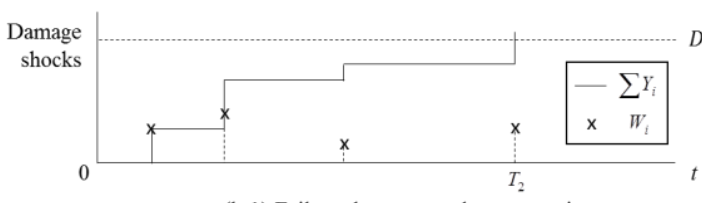

(b-1) Failure due to cumulate stagnation

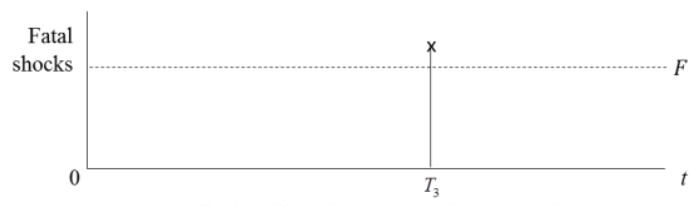

(b-2) Failure due to Immediate stagnation

Figure 11 Failure due to wear and stagnation

The three events can be modeled as below:

(1) Excessive wear

We assume the wear process can be described by a linear degradation path, $X(t)=\varphi+\beta t$, where $\varphi$ is a constant and $\beta$ is a random variable with $\beta \sim N\left(\mu_{\beta}, \sigma_{\beta}^{2}\right)$. Once $X(t)$ exceeds the degradation threshold $H$, the system will fail. 
(2) Immediate stagnation

Let $W_{i}$ be the $i$ th shock magnitude. The immediate stagnation happens immediately when $W_{N(t)}>F$, where $N(t)$ denotes the number of this fatal shock and $F$ denotes the fatal shock threshold. Thus the probability that the system survives the applied stress from the $i$ th shock is $P\left(W_{i}<F\right)=\Phi\left(\frac{F-\mu_{W}}{\sigma_{W}}\right)$.

(3) Cumulative stagnation

We assume that when $W_{i}<F$, the shock will cause a damage to the system. The damage size is denoted by $Y_{i} \mathrm{~s}$, which are i.i.d random variables, and $Y_{i} \sim N\left(\mu_{Y}, \sigma_{Y}^{2}\right)$. When $\sum Y_{i}$ exceeds the damage shock threshold $D$, the system will also fail.

Therefore, the reliability of the system at time $t$ should be:

$$
\begin{aligned}
R(t)= & P(X(t)<H, N(t)=0) \\
& +\sum_{i=1}^{\infty} P\left(X(t)<H, W_{1}<F, \cdots, W_{N(t)}<F, \sum_{j=1}^{N(t)} Y_{j}<D, N(t)=i\right) \\
= & P(X(t)<H, N(t)=0) \\
& +\sum_{i=1}^{\infty} P(X(t)<H) \cdot\left[P\left(W_{i}<F\right)\right]^{i} \cdot P\left(\sum_{j=1}^{N(t)} Y_{j}<D \mid N(t)=i\right) \cdot P(N(t)=i) \\
= & \sum_{i=0}^{\infty} \Phi\left(\frac{H-\mu_{\beta} t-\varphi}{\sigma_{\beta} t}\right) \cdot \Phi^{i}\left(\frac{F-\mu_{W}}{\sigma_{W}}\right) \cdot \Phi\left(\frac{D-i \mu_{Y}}{i \sigma_{Y}}\right) \cdot \frac{e^{-\lambda t}(\lambda t)^{i}}{i !} .
\end{aligned}
$$

Considering the influence of epistemic uncertainty, the 11 parameters in the reliability model are estimated by experts in the form of intervals and listed in Table 5. The DRSQP method in Figure 6 is applied to calculate the reliability box of the estimated reliability. The result is given in Figure 12. The value of $A_{R B}$ is also calculated using Algorithm 1, which is $3.6907 \times 10^{4}(\mathrm{~h})$.

The two-stage procedure developed in Section 4 is then applied to control the epistemic uncertainty. Suppose the required epistemic uncertainty is $A_{R B, r e q}=7.5 \times 10^{3} h$, and the cost function is the same as (25). In the first stage, an initial attempt is conducted using GA, whose result is used in the second stage as the initial values of the SQP. In the second stage, SQP method is used to improve the optimality of the solution. The optimal solutions from the two stages are tabulated in Table 6, and the resulted reliability box is given in Figure 12. Figure 12 shows that by properly allocate resources to reduce the epistemic uncertainty on each parameter, the epistemic uncertainty on the estimated reliability can be reduced as required. 
Table 5 Intervals of parameters given by experts

\begin{tabular}{|c|c|c|c|c|}
\hline \multicolumn{2}{|c|}{ Parameters } & \multirow{2}{*}{$\begin{array}{c}\text { Lower Limit } \\
4.7\end{array}$} & \multirow{2}{*}{$\begin{array}{c}\text { Upper Limit } \\
5.3\end{array}$} & \multirow{2}{*}{$\begin{array}{c}\text { Midpoint } \\
5\end{array}$} \\
\hline & $H(\mathrm{~mm})$ & & & \\
\hline & $F(m m)$ & 1.4 & 1.6 & 1.5 \\
\hline & $D(m m)$ & 7.0 & 8.0 & 7.5 \\
\hline & $\lambda$ & $2 \times 10^{-5}$ & $3 \times 10^{-5}$ & $2.5 \times 10^{-5}$ \\
\hline & $\varphi$ & $-5 \times 10^{-3}$ & $5 \times 10^{-3}$ & 0 \\
\hline \multirow{2}{*}{$\beta$} & $\mu_{\beta}(\mathrm{mm} / \mathrm{s})$ & $8 \times 10^{-5}$ & $1.2 \times 10^{-4}$ & $1 \times 10^{-4}$ \\
\hline & $\sigma_{\beta}(m m / s)$ & $8 \times 10^{-6}$ & $1.2 \times 10^{-5}$ & $1 \times 10^{-5}$ \\
\hline \multirow{2}{*}{ Y } & $\mu_{Y}(m m)$ & 1.1 & 1.3 & 1.2 \\
\hline & $\sigma_{Y}(m m)$ & 0.15 & 0.25 & 0.2 \\
\hline \multirow{2}{*}{ W } & $\mu_{W}(m m)$ & 1.1 & 1.3 & 1.2 \\
\hline & $\sigma_{W}(\mathrm{~mm})$ & 0.15 & 0.25 & 0.2 \\
\hline
\end{tabular}

2

Table 6 The optimal solutions from the two stages

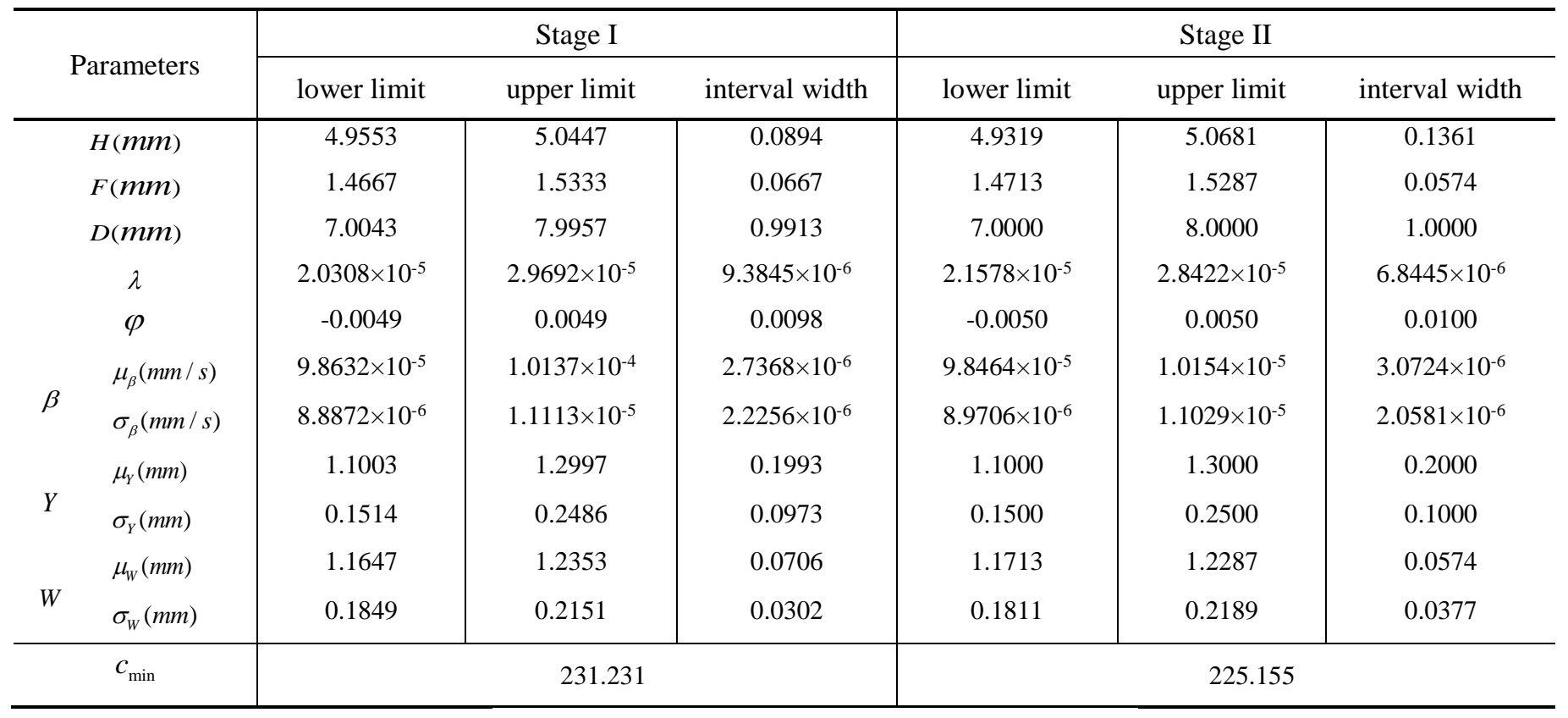

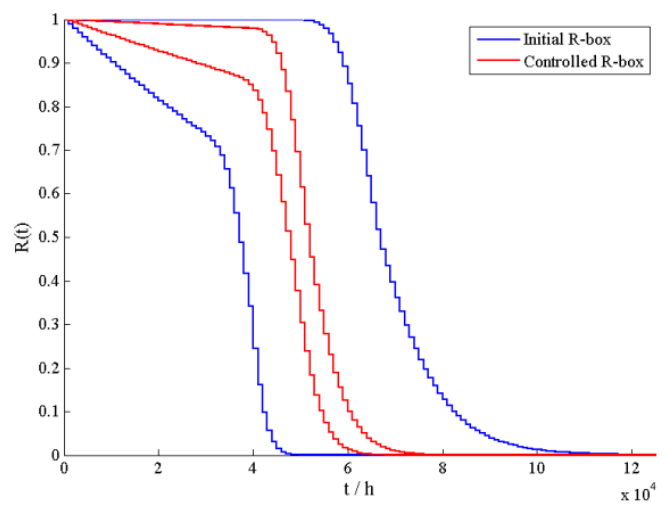

Figure 12 Reliability box of the sliding spool 


\section{Conclusions}

In this paper, probability box is introduced as a tool to describe the effect of epistemic uncertainty on the MDCFP model. To efficiently propagate epistemic uncertainty and construct the probability box, we developed a dimension-reduced SQP method. In addition, an optimization model is developed to allocate the resource in order to optimally reduce the effect of epistemic uncertainty. Two case studies demonstrated that the epistemic uncertainty in the MDCFP model can be satisfactorily described and controlled by the developed methods. A limitation of the DRSQP method is that the reliability model of the MDCFP must be second derivable and the derivative functions should be in explicit forms. Developing DRSQP method for generic reliability functions is an interesting but challenging future research topic. Also, the possibility of applying other theories to describe the epistemic uncertainty in the MDCFP models could also be explored, e.g., evidence theory, Bayesian theory, possibility theory, etc.

\section{Acknowledgements}

This work has been performed within the initiative of the Center for Resilience and Safety of Critical Infrastructures (CRESCI, http://cresci.buaa.edu.cn). This work is supported by Natural Science Foundation of China (NSFC) under contract No. 61573043.

\section{Appendix}

The gradients of parameters in (21) are shown as below:

$$
\frac{\partial R}{\partial H}=\sum_{i=0}^{\infty} P_{L}^{i} \phi\left(\frac{H-\left(\mu_{\beta} t_{0}+\varphi+i \mu_{Y}\right)}{\sqrt{\sigma_{\beta}^{2} t_{0}^{2}+i \sigma_{Y}^{2}}}\right) \frac{1}{\sqrt{\sigma_{\beta}^{2} t_{0}^{2}+i \sigma_{Y}^{2}}} \frac{e^{-\lambda t_{0}}\left(\lambda t_{0}\right)^{i}}{i !}>0
$$

$$
\frac{\partial R}{\partial D}=\sum_{i=0}^{\infty} \frac{i P_{L}^{i-1}}{\sigma_{W}} \phi\left(\frac{D-\mu_{W}}{\sigma_{W}}\right) \Phi\left(\frac{H-\left(\mu_{\beta} t_{0}+\varphi+i \mu_{Y}\right)}{\sqrt{\sigma_{\beta}^{2} t_{0}^{2}+i \sigma_{Y}^{2}}}\right) \frac{e^{-\lambda t_{0}}\left(\lambda t_{0}\right)^{i}}{i !}>0
$$$$
\frac{\partial R}{\partial \mu_{\beta}}=\sum_{i=0}^{\infty} P_{L}^{i} \phi\left(\frac{H-\left(\mu_{\beta} t_{0}+\varphi+i \mu_{Y}\right)}{\sqrt{\sigma_{\beta}^{2} t_{0}^{2}+i \sigma_{Y}^{2}}}\right) \frac{-t_{0}}{\sqrt{\sigma_{\beta}^{2} t_{0}^{2}+i \sigma_{Y}^{2}}} \frac{e^{-\lambda t_{0}}\left(\lambda t_{0}\right)^{i}}{i !}<0
$$

$$
\frac{\partial R}{\partial \mu_{Y}}=\sum_{i=0}^{\infty} P_{L}^{i} \phi\left(\frac{H-\left(\mu_{\beta} t_{0}+\varphi+i \mu_{Y}\right)}{\sqrt{\sigma_{\beta}^{2} t_{0}^{2}+i \sigma_{Y}^{2}}}\right) \frac{-i}{\sqrt{\sigma_{\beta}^{2} t_{0}^{2}+i \sigma_{Y}^{2}}} \frac{e^{-\lambda t_{0}}\left(\lambda t_{0}\right)^{i}}{i !}<0
$$

$$
\frac{\partial R}{\partial \mu_{W}}=-\sum_{i=0}^{\infty} \frac{i P_{L}^{i-1}}{\sigma_{W}} \phi\left(\frac{D-\mu_{W}}{\sigma_{W}}\right) \Phi\left(\frac{H-\left(\mu_{\beta} t_{0}+\varphi+i \mu_{Y}\right)}{\sqrt{\sigma_{\beta}^{2} t_{0}^{2}+i \sigma_{Y}^{2}}}\right) \frac{e^{-\lambda t_{0}}\left(\lambda t_{0}\right)^{i}}{i !}<0
$$




$$
\frac{\partial R}{\partial \sigma_{\beta}}=-\sum_{i=0}^{\infty} P_{L}^{i} t_{0}^{2} \sigma_{\beta} \phi\left(\frac{H-\left(\mu_{\beta} t_{0}+\varphi+i \mu_{Y}\right)}{\sqrt{\sigma_{\beta}^{2} t_{0}^{2}+i \sigma_{Y}^{2}}}\right)\left(H-\left(\mu_{\beta} t_{0}+\varphi+i \mu_{Y}\right)\right)\left(\sigma_{\beta}^{2} t_{0}^{2}+i \sigma_{Y}^{2}\right)^{-\frac{3}{2}} \frac{e^{-\lambda t_{0}}\left(\lambda t_{0}\right)^{i}}{i !},
$$

$$
\frac{\partial R}{\partial \sigma_{Y}}=-\sum_{i=0}^{\infty} P_{L}^{i} i \sigma_{Y} \phi\left(\frac{H-\left(\mu_{\beta} t_{0}+\varphi+i \mu_{Y}\right)}{\sqrt{\sigma_{\beta}^{2} t_{0}^{2}+i \sigma_{Y}^{2}}}\right)\left(H-\left(\mu_{\beta} t_{0}+\varphi+i \mu_{Y}\right)\right)\left(\sigma_{\beta}^{2} t_{0}^{2}+i \sigma_{Y}^{2}\right)^{-\frac{3}{2}} \frac{e^{-\lambda t_{0}}\left(\lambda t_{0}\right)^{i}}{i !}
$$

$$
\frac{\partial R}{\partial \lambda}=\sum_{i=0}^{\infty} P_{L}^{i} \Phi\left(\frac{H-\left(\mu_{\beta} t_{0}+\varphi+i \mu_{Y}\right)}{\sqrt{\sigma_{\beta}^{2} t_{0}^{2}+i \sigma_{Y}^{2}}}\right) \frac{e^{-\lambda t_{0}} t_{0}\left(\lambda t_{0}\right)^{i-1}\left(i-\lambda t_{0}\right)}{i !}
$$

\section{References}

[1] Q. Feng, D. W.Coit, Reliability modeling for multiple dependent competing failure processes: An MEMS application, International Journal of Performability Engineering, 6 (1): 100-102, 2010.

[2] S. Ghorbani, Reliability Analysis for Systems Subject to Degradation and Shocks, in, Rutgers University, New Jersey, 2014.

[3] H. Peng, Q. Feng, D.W. Coit, Reliability and maintenance modeling for systems subject to multiple dependent competing failure processes, IIE Transactions, 43 (1): 12-22, 2010.

[4] A.J. Lemoine, M.L. Wenocur, On Failure Modeling, Naval research logistics quarterly, 32 (3): 497-508, 1985.

[5] Y.P. Wang, H. Pham, A Multi-Objective Optimization of Imperfect Preventive Maintenance Policy for Dependent Competing Risk Systems With Hidden Failure, Ieee T Reliab, 60 (4): 770-781, 2011.

[6] W.J. Li, H. Pham, Reliability modeling of multi-state degraded systems with multi-competing failures and random shocks, Ieee T Reliab, 54 (2): 297-303, 2005.

[7] W.J. Li, H. Pham, An inspection-maintenance model for systems with multiple competing processes, Ieee T Reliab, 54 (2): 318-327, 2005.

[8] E. Keedy, Q.M. Feng, Reliability Analysis and Customized Preventive Maintenance Policies for Stents With Stochastic Dependent Competing Risk Processes, Ieee T Reliab, 62 (4): 887-897, 2013.

[9] Y.H. Lin, Y.F. Li, E. Zio, Integrating Random Shocks Into Multi-State Physics Models of Degradation Processes for Component Reliability Assessment, Ieee T Reliab, 64 (1): 154-166, 2015.

[10] A.D. Kiureghian, O. Ditlevsen, Aleatory or epistemic? Does it matter?, Structural Safety, 31 (2): 105-112, 2009.

[11] M.H. Faber, On the treatment of uncertainties and probabilities in engineering decision analysis, J Offshore Mech Arct, 127 (3): 243-248, 2005.

[12] J.C. Helton, J.D. Johnson, Quantification of margins and uncertainties: Alternative representations of epistemic uncertainty, Reliability Engineering \& System Safety, 96 (9): 1034-1052, 2011.

[13] H.-R. Bae, R.V. Grandhi, R.A. Canfield, An approximation approach for uncertainty quantification using evidence 
theory, Reliability Engineering \& System Safety, 86 (3): 215-225, 2004.

2 [14] D. Dubois, H. Prade, Possibility theroy, Plenum, New York, 1986.

3 [15] M. Beer, S. Ferson, V. Kreinovich, Imprecise probabilities in engineering analyses, Mechanical Systems and Signal Processing, 37 (1-2): 4-29, 2013.

[16] S. Ferson, L.R. Ginzburg, Different methods are needed to propagate ignorance and variability, Reliability Engineering \& System Safety, 54 (2-3): 133-144, 1996.

[17] T. Igusa, S.G. Buonopane, B.R. Ellingwood, Bayesian analysis of uncertainty for structural engineering applications, Structural Safety, 24 (2002): 165-186, 2002.

[18] M.C.M. Troffaes, G. Walter, D. Kelly, A robust Bayesian approach to modeling epistemic uncertainty in common-cause failure models, Reliability Engineering \& System Safety, 125: 13-21, 2014.

[19] R. Flage, P. Baraldi, E. Zio, T. Aven, Probability and possibility-based representations of uncertainty in fault tree analysis, Risk Anal, 33 (1): 121-133, 2013.

[20] J. Rohmer, C. Baudrit, The use of the possibility theory to investigate the epistemic uncertainties within scenario-based earthquake risk assessments, Natural Hazards, 56 (3): 613-632, 2010.

[21] D.R. Karanki, H.S. Kushwaha, A.K. Verma, S. Ajit, Uncertainty Analysis Based on Probability Bounds (P-Box) Approach in Probabilistic Safety Assessment, Risk Anal, 29 (5): 662-675, 2009.

[22] H. Zhang, R.L. Mullen, R.L. Muhanna, Interval Monte Carlo methods for structural reliability, Structural Safety, 32 (3): 183-190, 2010.

[23] J.M. Aughenbaugh, C.J.J. Paredis, Probability bounds analysis as a general approach to sensitivity analysis in decision making under uncertainty, in: SAE2007 Transactions Journal of Passenger Cars: Mechanical Systems, SAE International, Warrendale, Pennsylvania, 2007, pp. 1325-1339.

[24] L. Goldwasser, L. Ginzburg, S. Ferson, Quantitative Methods for Conservation Biology, Springer-Berlag, New York, 2000.

[25] E. Kriegler, H. Held, Utilizing belief functions for the estimation of future climate change, Int J Approx Reason, 39 (2-3): 185-209, 2005

[26] P. Walley, T.L. Fine, Towards a frequentist theory of upper and lower probability, The Annals of Statistics, 10 (3): 741-761, 1982.

[27] M. Wolfenson, T.L. Fine, Bayes-like decision making with upper and lower probabilities, Journal of the American Statistical Association, 77 (377): 80-88, 1982.

[28] N.-C. Xiao, H.-Z. Huang, Z. Wang, Y. Pang, L. He, Reliability sensitivity analysis for structural systems in interval probability form, Structural and Multidisciplinary Optimization, 44 (5): 691-705, 2011.

[29] L.G. Crespo, S.P. Kenny, D.P. Giesy, Reliability analysis of polynomial systems subject to p-box uncertainties, Mechanical Systems and Signal Processing, 37 (1-2): 121-136, 2013.

[30] H. Zhang, Interval importance sampling method for finite element-based structural reliability assessment under parameter uncertainties, Structural Safety, 38: 1-10, 2012.

[31] H. Zhang, H. Dai, M. Beer, W. Wang, Structural reliability analysis on the basis of small samples: An interval quasi-Monte Carlo method, Mechanical Systems and Signal Processing, 37 (1-2): 137-151, 2013.

[32] X. Yang, Y. Liu, Y. Zhang, Z. Yue, Hybrid reliability analysis with both random and probability-box variables, Acta Mechanica, 226 (5): 1341-1357, 2014.

[33] F. Tonon, Using random set theory to propagate epistemic uncertainty through a mechanical system, Reliability 
Engineering \& System Safety, 85 (1-3): 169-181, 2004.

2 [34] Reliability prediction handbook for electronic equipment, in, Electronic Information Department, Beijing, 2006.

3 [35] G. Li, Numerical Optimization Methods, Peking University, Beijing, 2014.

4 [36] S.P. Han, A globally convergent method for nonlinear programming, Journal of Optimization Theory and Applications, $522(3): 297-309,1977$.

6 [37] H. Aytug, M. Khouja, F.E. Vergara, Use of genetic algorithms to solve production and operations management

8 [38] X. Liao, Research on the wear mechanism and life modeling method of aero-hydraulic spool valve, in, Beihang 9 University, Beijing, 2014.

10 [39] M. Fan, Z. Zeng, Z. Enrico, R. Kang, Modeling dependent competing failure processes with degradation-shock 11 dependence, Ieee T Reliab, (Under Review), 2016.

12 [40] Z. Zhou, Research on mechanism of contaminant lock for hydraulic valve, Chinese Hydraulics \& Pneumatics, 1 13 (1994): 15-17, 1994. 